\title{
A Central Limit Theorem for the Fourth Wick Power of the Free Lattice Field
}

\author{
S. Albeverio ${ }^{1,2}$, X.Y. Zhou ${ }^{1,3,4}$
}

\author{
${ }^{1}$ Institute of Mathematics, Ruhr-University Bochum, D-44780 Bochum, Germany. \\ E-mail: sergio albeverio@rz ruhr-uni-bochum de \\ ${ }^{2}$ BiBoS; SFB 237 Bochum-Essen-Düsseldorf; CERFIM, Locarno \\ 3 Department of Mathematics, University of Bielefeld, D-33501 Bielefeld, Germany \\ ${ }^{4}$ Department of Mathematics, Beijing Normal University, Beijing 100875, China
}

Received: 1 October 1995/Accepted: 23 January 1996

Abstract: Let $G_{a}$ be the free lattice field measure of mass $m_{0}$ on $a Z^{d}$, and : $\phi_{x}^{4}:$ be the corresponding fourth Wick power of the lattice field $\phi_{x}$. Let $g \in C_{0}\left(R^{d}\right), g \geqq 0$, be a given function and $a^{\prime}=a^{\prime}(a) \geqq a$ satisfy: $\lim _{a \rightarrow 0^{+}} a^{\prime}=0$ and $a^{\prime} Z^{d} \subset a Z^{d}$. We prove that if $d \geqq 3$, or $d=2$ and $\lim _{a \rightarrow 0^{+}} a^{\prime}|\log a|^{2}=\infty$, then $\left\{a^{\prime d} \sum_{x \in a^{\prime} Z^{d}} g_{x}\right.$ $\left.: \phi_{x}^{4}:\right\}$ satisfies the central limit theorem: there is $V\left(a, a^{\prime}\right)$ with $\lim _{a \rightarrow 0^{+}} V\left(a, a^{\prime}\right)=$ $\infty$ such that the distribution of $V\left(a, a^{\prime}\right)^{-1} a^{\prime d} \sum_{x \in a^{\prime} Z^{d}} g_{x}: \phi_{x}^{4}:$ under $G_{a}$ is convergent to the standard normal distribution, as $a \rightarrow 0^{+}$.

\section{Introduction}

Let $G_{a}$ be the free lattice field measure of mass $m_{0}>0$ and lattice spacing $a>0$ on $a Z^{d}=\left\{a x: x \in Z^{d}\right\}$, and let $\langle\cdot\rangle_{G_{a}}$ denote the expectation with respect to $G_{a}$. Let

$$
C^{(a)}(x-y)=\left\langle\phi_{x} \phi_{y}\right\rangle_{G_{a}}
$$

$G_{a}$ is thus the (lattice) Gaussian measure with covariance $C^{(a)}$. It is easy to show that (see $[\mathrm{Si}, \mathrm{BFS}]$ )

$$
C^{(a)}(x-y)=(2 \pi)^{-d} \int_{\left[-\frac{\pi}{a}, \frac{\pi}{a}\right]^{d}}\left[m_{0}^{2}+2 a^{-2} \sum_{j=1}^{d}\left(1-\cos a k_{j}\right)\right]^{-1} e^{i k \cdot(x-y)} d k,
$$

with $k=\left(k_{1}, \ldots, k_{d}\right)$. Let $: \phi_{x}^{4}:$ be the fourth Wick order of $\phi_{x}$, i.e.

$$
: \phi_{x}^{4}:=\phi_{x}^{4}-6 \phi_{x}^{2}\left\langle\phi_{x}^{2}\right\rangle_{G_{a}}+3\left\langle\phi_{x}^{2}\right\rangle_{G_{a}}^{2} .
$$

Let $g(\geqq 0) \in C_{0}\left(R^{d}\right)$ be a given function and $a^{\prime}=a^{\prime}(a)$ satisfy: $a^{\prime} Z^{d} \subset a Z^{d}$ and $\lim _{a \rightarrow 0^{+}} a^{\prime}=0$. From this we can see that $a^{\prime} \geqq a$. For simplicity we also assume that $\lim _{a \rightarrow 0^{+}} \frac{a}{a^{\prime}}$ exists. The main aim of this paper is to discuss conditions on $a$ and $a^{\prime}$ such that the central limit theorem holds for the system $\left\{\xi\left(a, a^{\prime}\right)\right\}$, where

$$
\xi\left(a, a^{\prime}\right)=a^{\prime d} \sum_{x \in a^{\prime} Z^{d}} g_{x}: \phi_{x}^{4}:
$$


The central limit theorem for other systems of statistical mechanics has been discussed by many authors (see e.g. [Ne] and references therein). A very general central limit theorem for FKG systems was obtained in [Ne]. However, our system $\left\{\xi\left(a, a^{\prime}\right)\right\}$, with underlying probability measure $G_{a}$, does not seem to be a FKG system.

Let

$$
V\left(a, a^{\prime}\right)=\left\langle\left(a^{\prime d} \sum_{x \in a^{\prime} Z^{d}} g_{x}: \phi_{x}^{4}:\right)^{2}\right\rangle_{G_{a}}^{1 / 2} .
$$

For $d=3$ and $a=a^{\prime}$ we have $\lim _{a \rightarrow 0^{+}} V\left(a, a^{\prime}\right)=\infty$. From [P, Theorem 1.1] and $[\mathrm{Fe}$, Theorem 1] we can see that

$$
\lim _{a \rightarrow 0^{+}}\left\langle\exp \left(-\frac{\lambda}{V(a, a)} \xi(a, a)+\frac{\lambda^{2}}{2}\right)\right\rangle_{G_{a}}=1, \quad \forall \lambda \in[0, \infty) .
$$

Using this we can easily show that the system $\{\xi(a, a)\}$ for $d=3$ satisfies the central limit theorem, i.e.

$$
\lim _{a \rightarrow 0^{+}} G_{a}\left(\frac{\xi(a, a)}{V(a, a)} \leqq x\right)=(2 \pi)^{-1 / 2} \int_{-\infty}^{x} e^{-\frac{y^{2}}{2}} d y, \quad \forall x \in R .
$$

For convenience, we will denote the property (1.2) by the following:

$$
\frac{\xi(a, a)}{V(a, a)} \stackrel{\mathscr{D}}{\longrightarrow} N(0,1), \quad a \rightarrow 0^{+} .
$$

However, the proof of (1.1) given in [P] or [Fe] (based on an idea from [GJ]) is very complicated. In this paper we will use a different approach to prove a general result which contains (1.2) as a particular case.

Our main results are as follows (we always assume $\lim _{a \rightarrow 0^{+}} a^{\prime}=0$ ).

Theorem 1.1. Suppose that $g(\geqq 0) \in C_{0}\left(R^{d}\right)$ and $\int_{R^{d}} g_{x}^{2} d x>0$.

(i) For $d=2$, if $\lim _{a \rightarrow 0^{+}} a^{\prime}|\log a|^{2}=\infty$, then

$$
\frac{\xi\left(a, a^{\prime}\right)}{V\left(a, a^{\prime}\right)} \stackrel{\mathscr{D}}{\longrightarrow} N(0,1), \quad a \rightarrow 0^{+}
$$

where $V\left(a, a^{\prime}\right)$ satisfies the following property:

$$
\lim _{a \rightarrow 0^{+}} \frac{V\left(a, a^{\prime}\right)^{2}}{a^{\prime} C^{(a)}(0)^{2}}=4 ! \int_{R^{2}} g_{x}^{2} d x
$$

(ii) For $d \geqq 3$, (1.3) is always true, where $V\left(a, a^{\prime}\right)$ satisfies:

$$
\lim _{a \rightarrow 0^{+}} V\left(a, a^{\prime}\right)^{-2} 4 ! a^{\prime 2 d} \sum_{x, y \in a^{\prime} Z^{d}} g_{x} g_{y} C^{(a)}(x-y)^{4}=1
$$

More precisely, there exists a constant $c_{d} \in(0, \infty)$ such that

$$
\lim _{a \rightarrow 0^{+}} \frac{V\left(a, a^{\prime}\right)^{2}}{a^{\prime d} a^{-4(d-2)}}=c_{d} .
$$


Note. We remark that there is a constant $c \in(0, \infty)$ such that

$$
C^{(a)}(0) \geqq \begin{cases}c a^{-(d-2)}, & d \geqq 3, \\ c|\log a|, & d=2 .\end{cases}
$$

Hence, if $d \geqq 3$ and $a^{\prime} \geqq a$, or $d=2$ and $\lim _{a \rightarrow 0^{+}} a^{\prime}|\log a|^{2}=\infty$, then

$$
\lim _{a \rightarrow 0^{+}} V\left(a, a^{\prime}\right)=\infty .
$$

The proof of Theorem 1.1 will be given in the next section. In Sect. 3 below we will prove the key lemma (i.e. Lemma 2.1 below).

At the end of this section we give a remark on Theorem 1.1 above. For this purpose we introduce some notations. Let $\mu_{0}$ be the Gaussian measure on $\mathscr{S}^{\prime}\left(R^{d}\right)$ with the covariance $C=\left(-\Delta+m_{0}^{2}\right)^{-1}$. Let $\mu^{-1}(k)=\left(|k|^{2}+m_{0}^{2}\right)^{-1}$ and

$$
\mu_{a}^{-1}(k)=\left(m_{0}^{2}+2 a^{-2} \sum_{j=1}^{d}\left(1-\cos \left(a k_{j}\right)\right)\right)^{-1}
$$

where $k=\left(k_{1}, \ldots, k_{d}\right)$ and $|k|^{2}=k_{1}^{2}+\cdots+k_{d}^{2}$. Let $f_{x, a}(\cdot)$ be the function whose Fourier transform is

$$
\hat{f}_{x, a}(k)= \begin{cases}(2 \pi)^{-d / 2} e^{-i k x} \mu_{a}(k)^{-1} \mu(k), & \text { if }\left|k_{j}\right| \leqq \pi / a, j=1, \ldots, d, \\ 0, & \text { otherwise }\end{cases}
$$

It is easy to check that (see e.g. [Si, Sect. VIII.1])

$$
\left\langle\phi\left(f_{x, a}\right) \phi\left(f_{y, a}\right)\right\rangle_{\mu_{0}}=\left\langle\phi_{x} \phi_{y}\right\rangle_{G_{a}} .
$$

In this sense we can realize the Gaussian field $\phi_{x}$ on $a Z^{d}$ by $\phi_{x}=\phi\left(f_{x, a}\right)$, which is defined on $\mathscr{S}^{\prime}\left(R^{d}\right)$.

The central limit theorem given in Theorem 1.1 is probably not enough for proving the existence of the continuum limit of some lattice $\phi^{4}$-fields with counterterms. However, it can help us to understand the form of some counterterm, and moreover it might be used to study some properties of continuum $\phi^{4}$-fields like the $\phi_{3}^{4}$-quantum field constructed in [BFS] or [P] and the new continuum $\phi_{2}^{4}$-field constructed in [AZ]. Heuristically, from Theorem 1.1 (ii) above we can see that the continuum $\phi_{3}^{4}$-field with a spatial cutoff constructed in [BFS] or in [P] is singular with respect to the Gaussian measure $\mu_{0}$ on $\mathscr{S}^{\prime}\left(R^{3}\right)$. Likewise, by Theorem 1.1 (i) we believe that the continuum $\phi_{2}^{4}$-field $\mu_{\lambda, g}$ (see [AZ]) in the case where $\lim _{a \rightarrow 0^{+}} a^{\prime}|\log a|^{2}=\infty$ is singular with respect to the Gaussian measure $\mu_{0}$ on $\mathscr{S}^{\prime}\left(R^{2}\right)$.

\section{Proof of the Main Result}

Let us first recall some properties of the Wick powers (see [Si, Sect. I.1]). If $\phi_{x}$ is a Gaussian random variable (under a Gaussian measure $G$ ), then

$$
: \exp \left(t \phi_{x}\right):=\exp \left(t \phi_{x}-\frac{1}{2} t^{2}\left\langle\phi_{x}^{2}\right\rangle_{G}\right)
$$


which implies

$$
\begin{aligned}
\prod_{i=1}^{n}: \exp \left(t_{i} \phi_{x_{l}}\right) & :=\exp \left(\sum_{i=1}^{n} t_{i} \phi_{x_{i}}\right) \exp \left(-\frac{1}{2} \sum_{i=1}^{n} t_{i}^{2}\left\langle\phi_{x_{i}}^{2}\right\rangle_{G}\right) \\
& =: \exp \left(\sum_{i=1}^{n} t_{i} \phi_{x_{l}}\right): \exp \left(\sum_{1 \leqq i<j \leqq n} t_{i} t_{j}\left\langle\phi_{x_{l}} \phi_{x_{J}}\right\rangle_{G}\right) .
\end{aligned}
$$

We remark that $\exp \left(\sum_{1 \leqq i<j \leqq n} t_{i} t_{j}\left\langle\phi_{x_{i}} \phi_{x_{j}}\right\rangle_{G}\right)$ is not random and

$$
\left\langle: \exp \left(\sum_{i=1}^{n} t_{i} \phi_{x_{l}}\right):\right\rangle_{G}=1 \text {. }
$$

Therefore,

$$
\left\langle\prod_{i=1}^{n}: \exp \left(t_{i} \phi_{x_{l}}\right):\right\rangle_{G}=\exp \left(\sum_{1 \leqq i<j \leqq n} t_{i} t_{j}\left\langle\phi_{x_{l}} \phi_{x_{j}}\right\rangle_{G}\right) .
$$

From (2.1) we can see that

$$
V\left(a, a^{\prime}\right)^{2}=4 ! a^{2 d} \sum_{x, y \in a^{\prime} Z^{d}} g_{x} g_{y}\left\langle\phi_{x} \phi_{y}\right\rangle_{G_{a}}^{4}
$$

In this section we always assume that $g \geqq 0$ is a given function in $C_{0}\left(R^{d}\right)$ and $\int_{R^{d}} g_{x}^{2} d x>0$. Let $\eta(a)=V\left(a, a^{\prime}\right)^{-1 / 2} \xi\left(a, a^{\prime}\right)$. To prove (1.3) we only need to prove that

$$
\begin{gathered}
\lim _{a \rightarrow 0^{+}}\left\langle\eta(a)^{2^{k}}\right\rangle_{G_{a}}=(2 k-1) ! !, \quad k=1,2, \ldots, \\
\lim _{a \rightarrow 0^{+}}\left\langle\eta(a)^{2 k+1}\right\rangle_{G_{a}}=0, \quad k=1,2, \ldots,
\end{gathered}
$$

where $(2 k-1) ! !=(2 k-1) \cdot(2 k-3) \cdots 3 \cdot 1$ for $k \geqq 1$. To do so we need to compute the quantity $\left\langle\xi\left(a, a^{\prime}\right)^{k}\right\rangle_{G_{a}}$. We remark that

$$
\left\langle\xi\left(a, a^{\prime}\right)^{k}\right\rangle_{G_{a}}=a^{2 k} \sum_{x_{1},, x_{k} \in a^{\prime} Z^{2}} g_{x_{1}} \cdots g_{x_{k}}\left\langle: \phi_{x_{1}}^{4}: \cdots: \phi_{x_{k}}^{4}:\right\rangle_{G_{a}} .
$$

Thus, it suffices to compute $\left\langle: \phi_{x_{1}}^{4}: \cdots: \phi_{x_{k}}^{4}:\right\rangle_{G_{a}}$. For this purpose we need to compare the terms with the coefficient $\prod_{i=1}^{k} t_{i}^{4}$ on both sides of (2.1). In general, the term with the coefficient $\prod_{i=1}^{k} t_{i}^{4}$ on the right-hand side of (2.1) can be expressed as a sum of some quantities of the following form:

$$
\begin{aligned}
q_{a, a^{\prime}}\left(x_{1}, \ldots, x_{k}\right):=\mathscr{C}_{k} \prod_{j=2}^{k}\left\langle\phi_{x_{1}} \phi_{x_{j}}\right\rangle_{G_{a}}^{l_{1, j}} \prod_{j=3}^{k}\left\langle\phi_{x_{2}} \phi_{x_{j}}\right\rangle_{G_{a}}^{l_{2, j}} \\
\ldots \prod_{j=k-1}^{k}\left\langle\phi_{x_{k-2}} \phi_{x_{j}}\right\rangle_{G_{a}}^{l_{k-2, j}}\left\langle\phi_{x_{k-1}} \phi_{x_{k}}\right\rangle_{G_{a}}^{l_{k-1, k}},
\end{aligned}
$$

where $\mathscr{C}_{k}$ is just the coefficient, $l_{i, j} \in\{0,1,2,3,4\}, \sum_{j=2}^{k} l_{1, j}=4$ and

$$
\sum_{j=1}^{i-1} l_{j, i}+\sum_{j=i+1}^{k} l_{i, j}=4, \quad i=2, \ldots, k-1 .
$$


We remark that there is a one to one correspondence between the quantity $q_{a, a^{\prime}}$ and the set of $l_{i, j}$. For convenience we will use the set of $l_{i, j}$ to represent the quantity $q_{a, a^{\prime}}$.

Let

$$
Q_{a, a^{\prime}}\left(x_{1}, \ldots, x_{k}\right)=\prod_{i=1}^{k} g_{x_{i}} \sum_{l_{i, j} \neq 4} q_{a, a^{\prime}}\left(x_{1}, \ldots, x_{k}\right),
$$

where the sum is taken over all $l_{i, j} \leqq 4$, in which there exists at least one pair $(i, j)$ such that $l_{i, j} \in\{1,2,3\}$. Let

$$
P_{a, a^{\prime}}\left(x_{1}, \ldots, x_{2 k}\right)=\prod_{j=1}^{2 k} g_{x_{j}} \sum_{l_{i, j}=4} q_{a, a^{\prime}}\left(x_{1}, \ldots, x_{2 k}\right),
$$

where the sum is taken over all $l_{i, j}$ with $l_{i, j}=4$. In this case (i.e. $l_{i, j}=4$ for all possible pairs $(i, j))$, the quantity $q_{a, a^{\prime}}\left(x_{1}, \ldots, x_{2 k}\right)$ can be expressed as follows:

$$
(4 !)^{k} \prod_{j=0}^{k-1}\left\langle\phi_{x_{i_{2}}} \phi_{x_{i_{2 j}+1}}\right\rangle_{G_{a}}^{4},
$$

which corresponds to the set $\left\{l_{i_{0}, i_{1}}=l_{i_{2}, i_{3}}=\cdots=l_{i_{2 k-2}, i_{2 k-1}}=4\right\}$, where $i_{0}=1$, and $\left\{i_{1}, \ldots, i_{2 k-1}\right\}=\{2, \ldots 2 k\}$. In fact we can derive the following expression:

$$
\begin{array}{r}
P_{a, a^{\prime}}\left(x_{1}, \ldots, x_{2 k}\right)=(4 !)^{k} \sum_{i_{1}}^{2 k} g_{x_{1}} g_{x_{i_{1}}}\left\langle\phi_{x_{1}} \phi_{x_{i_{1}}}\right\rangle_{G_{a}}^{4} \sum_{i_{3} \in\{2,, 2 k\} \backslash\left\{i_{2}, i_{3}\right\}} g_{x_{l_{2}}} g_{x_{i_{3}}}\left\langle\phi_{x_{i_{2}}} \phi_{x_{i_{3}}}\right\rangle_{G_{a}}^{4} \\
\cdots \sum_{i_{2 k-1} \in\{2,, 2 k\} \backslash\left\{i_{1}, i_{2 k-2}\right\}} g_{x_{i_{2 k-2}}} g_{x_{i_{2 k-1}}}\left\langle\phi_{x_{i_{2 k-2}}} \phi_{x_{i_{2 k-1}}}\right\rangle_{G_{a}}^{4},
\end{array}
$$

where $i_{2}, i_{4}, \ldots, i_{2 k-2}$ are defined in the following way:

$$
\begin{aligned}
& i_{2}=\min \left\{j: j \in\{2, \ldots, 2 k\} \backslash\left\{i_{1}\right\}\right\}, \\
& i_{2 l}=\min \left\{j: j \in\{2, \ldots, 2 k\} \backslash\left\{i_{1}, \ldots, i_{2 l-1}\right\}\right\}, \quad l=2, \ldots, k-1 .
\end{aligned}
$$

It is easy to see that there are $(2 k-1)$ !! choices of such kinds of sets: $\left\{i_{0}, i_{1}, \ldots\right.$, $\left.i_{2 k-2}, i_{2 k-1}\right\}$. Thus we obtain that

$$
\begin{aligned}
a^{\prime 2 k d} \sum_{x_{1},, x_{2 k} \in a^{\prime} Z^{d}} P_{a, a^{\prime}}\left(x_{1}, \ldots, x_{2 k}\right) & =(2 k-1) ! !\left(4 ! a^{\prime 2 d} \sum_{x, y \in a^{\prime} Z^{d}} g_{x} g_{y}\left\langle\phi_{x} \phi_{y}\right\rangle_{G_{a}}^{4}\right)^{k} \\
& =(2 k-1) ! ! V\left(a, a^{\prime}\right)^{2 k} .
\end{aligned}
$$

Hence, to prove (2.2) we only need to prove that

$$
\lim _{a \rightarrow 0^{+}} V\left(a, a^{\prime}\right)^{-2 k} a^{\prime 2 d k} \sum_{x_{1},, x_{2 k} \in a^{\prime} Z^{d}} Q_{a, a^{\prime}}\left(x_{1}, \ldots, x_{2 k}\right)=0, \quad k \geqq 1 .
$$

To this end let us first state a lemma.

Lemma 2.1. Under the same assumptions as in Theorem 1.1, the following is always true

$$
\lim _{a \rightarrow 0^{+}} V\left(a, a^{\prime}\right)^{-k} a^{\prime d k} \sum_{x_{1},, x_{k} \in a^{\prime} Z^{d}} Q_{a, a^{\prime}}\left(x_{1}, \ldots, x_{k}\right)=0
$$

for any $k \geqq 1$. 
This lemma will be proven in the next section.

Using Lemma 2.1 we can easily prove Theorem 1.1 .

Proof of Theorem 1.1. It is clear that (2.4) is an immediate consequence of Lemma 2.1. If $k=2 l+1$ for some $l \geqq 1$, then for each quantity $q_{a, a^{\prime}}\left(x_{1}, \ldots, x_{2 l+1}\right)$ there exists a pair $(i, j)$ such that $l_{i, j} \in\{1,2,3\}$. From this remark and (2.1) we can see that there is a constant $c=c(l)$ such that

$$
\left\langle\xi\left(a, a^{\prime}\right)^{2 l+1}\right\rangle_{G_{a}} \leqq c a^{\prime d(2 l+1)} \sum_{x_{1},, x_{2 l+1} \in a^{\prime} Z^{d}} Q_{a, a^{\prime}}\left(x_{1}, \ldots, x_{2 l+1}\right) .
$$

By Lemma 2.1 we then know that

$$
\lim _{a \rightarrow 0^{+}} V\left(a, a^{\prime}\right)^{-2 l-1}\left\langle\xi\left(a, a^{\prime}\right)^{2 l+1}\right\rangle_{G_{a}}=0,
$$

which proves (2.3). The proof of (1.3) is then complete.

Finally, let us discuss the asymptotic behaviour of $V\left(a, a^{\prime}\right)$ as $a \rightarrow 0^{+}$. Let

$$
\begin{aligned}
& I_{1}\left(a, a^{\prime}\right)=4 ! a^{\prime d} C^{(a)}(0)^{4}\left(a^{\prime d} \sum_{x \in a^{\prime} Z^{d}} g_{x}^{2}\right), \\
& I_{2}\left(a, a^{\prime}\right)=4 ! a^{\prime 2 d} \sum_{x, y \in a^{\prime} Z^{d}, x \neq y} g_{x} g_{y}\left\langle\phi_{x} \phi_{y}\right\rangle_{G_{a}}^{4} .
\end{aligned}
$$

By definition and (2.1) we know that

$$
V\left(a, a^{\prime}\right)^{2}=4 ! a^{\prime 2 d} \sum_{x, y \in a^{\prime} Z^{d}} g_{x} g_{y} C^{(a)}(x-y)^{4}=I_{1}+I_{2},
$$

where $C^{(a)}(x-y)=\left\langle\phi_{x} \phi_{y}\right\rangle_{G_{a}}$.

If $d=2$ and $\lim _{a \rightarrow 0^{+}} a^{\prime}|\log a|^{2}=\infty$, then $\quad \lim \sup _{a \rightarrow 0^{+}} I_{2}\left(a, a^{\prime}\right)<\infty$, $\lim _{a \rightarrow 0^{+}} a^{2} C^{(a)}(0)^{4}=\infty$ and

$$
\lim _{a \rightarrow 0^{+}} \frac{I_{1}\left(a, a^{\prime}\right)}{a^{\prime 2} C^{(a)}(0)^{4}}=4 ! \int_{R^{2}} g_{x}^{2} d x \in(0, \infty) .
$$

Hence, if $d=2$ and $\lim _{a \rightarrow 0^{+}} a^{\prime}|\log a|^{2}=\infty$, we have

$$
\lim _{a \rightarrow 0^{+}} \frac{V\left(a, a^{\prime}\right)^{2}}{a^{\prime 2} C^{(a)}(0)^{4}}=4 ! \int_{R^{2}} g_{x}^{2} d x \in(0, \infty) .
$$

If $d \geqq 3$ and $a^{\prime} \geqq a$ with $\lim _{a \rightarrow 0^{+}} a^{\prime}=0$, then there is a constant $k_{d} \in(0, \infty)$ such that

$$
\lim _{|x-y| \rightarrow 0, x, y \in a^{\prime} Z^{d}} \frac{C^{(a)}(x-y)}{(a \vee|x-y|)^{-(d-2)}}=k_{d} .
$$

Using this we can show that if $\lim _{a \rightarrow 0^{+}} x_{a}=x \in R^{2}$ with $x_{a} \in a Z^{d}$, then the following limit:

$$
\lim _{a \rightarrow 0^{+}} \frac{a^{d} \sum_{y \in a Z^{d}} g_{y} C^{(a)}\left(x_{a}-y\right)^{4}}{a^{d} a^{-4(d-2)}}
$$

exists. We denote this limit by $f_{x}$. It is easy to see that the function $f$ is positive 
and finite. If $\lim _{a \rightarrow 0^{+}} \frac{a}{a^{\prime}}:=\alpha \in(0,1]$, then we can also show that the limit

$$
\lim _{a \rightarrow 0^{+}} \frac{a^{\prime d} \sum_{y \in a^{\prime} Z^{d}, y \neq x_{a}} g_{y} C^{(a)}\left(x_{a}-y\right)^{4}}{a^{d} a^{-4(d-2)}}
$$

exists and is equal to $f_{x}$. In this case (i.e. $\alpha>0$ ), we have

$$
\lim _{a \rightarrow 0^{+}} \frac{V\left(a, a^{\prime}\right)^{2}}{a^{\prime d} a^{-4(d-2)}}=4 ! \alpha^{d} \int_{R^{2}} g_{x} f_{x} d x+4 ! \int_{R^{2}} g_{x}^{2} d x,
$$

which is positive and finite.

We remark that if $d \geqq 3$ and $a^{\prime} \geqq a$, then

$$
a^{\prime d} \sum_{y \in a^{\prime} Z^{d}, y \neq x} g_{y} C^{(a)}(x-y)^{4} \leqq O(1) a^{d} a^{-4(d-2)}, \quad \forall x \in a^{\prime} Z^{d}
$$

Hence, if $\alpha=0$, then

$$
\lim _{a \rightarrow 0^{+}} \frac{a^{\prime d} \sum_{y \in a^{\prime} Z^{d}, y \neq x_{a}} g_{y} C^{(a)}\left(x_{a}-y\right)^{4}}{a^{\prime d} a^{-4(d-2)}}=0 .
$$

In this case (i.e. $\alpha=0$ ), we have

$$
\lim _{a \rightarrow 0^{+}} \frac{V\left(a, a^{\prime}\right)^{2}}{a^{\prime d} a^{-4(d-2)}}=4 ! \int_{R^{2}} g_{x}^{2} d x
$$

The proof of Theorem 1.1 is then complete, provided Lemma 2.1 has been proved.

\section{Proof of Lemma 2.1}

The aim of this section is to complete the proof of Lemma 2.1. Let us first prove two lemmas.

Lemma 3.1. (i) There is a constant $c_{1}(d) \in(0, \infty)$ such that

$$
a^{\prime 2 d} \sum_{x, y \in a^{\prime} Z^{d}} g_{x} g_{y} C^{(a)}(x-y)^{2} \leqq \begin{cases}c_{1}(d)\left(a^{\prime d} C^{(a)}(0)^{2}+1\right), & d \geqq 2, d \neq 4, \\ c_{1}(4)\left(a^{\prime 4} C^{(a)}(0)^{2}+|\log a|\right), & d=4 .\end{cases}
$$

(ii) There is a constant $c_{2}(d) \in(0, \infty)$ such that

$$
a^{\prime 2 d} \sum_{x, y \in a^{\prime} Z^{d}} g_{x} g_{y} C^{(a)}(x-y) \leqq c_{2}(d) a^{\prime d} C^{(a)}(0), \quad d \geqq 2 .
$$

Proof. We only prove (i), since (ii) can be proven by a similar argument. It is obvious that

$$
a^{\prime 2 d} \sum_{x \in a^{\prime} Z^{d}} g_{x}^{2} C^{(a)}(0)^{2} \leqq O(1) a^{\prime d} C^{(a)}(0)^{2}
$$


Hence, we only need to provide a suitable upper bound for the following quantity:

$$
a^{\prime 2 d} \sum_{x, y \in a^{\prime} Z^{d}, x \neq y} g_{x} g_{y} C^{(a)}(x-y)^{2},
$$

which is denoted by $f_{1}\left(a, a^{\prime}\right)$. Since $a \leqq a^{\prime}$ and $\lim _{a \rightarrow 0^{+}} a^{\prime}=0$, by computation we show that

$$
f_{1}\left(a, a^{\prime}\right) \leqq O(1) f_{1}(a, a) .
$$

We recall again that

$$
C^{(a)}(x-y) \leqq \begin{cases}O(1)|\log | x-y||, & d=2, \\ O(1)|x-y|^{-d+2}, & d \geqq 3\end{cases}
$$

for $x, y \in a^{\prime} Z^{d}$ with $x \neq y$. Using this we can show that

$$
f_{1}(a, a) \leqq O(1), \quad d=2,3,
$$

and

$$
\begin{aligned}
f_{1}(a, a) & \leqq O(1) \max _{x \in a Z^{d}} a^{d} \sum_{y \in a Z^{d}} g_{y}|x-y|^{-2(d-2)} \\
& \leqq O(1) a^{-d+4} \leqq O(1) a^{\prime d} C^{(a)}(0)^{2}, \quad d \geqq 5 .
\end{aligned}
$$

Thus we have proven (i) for $d \geqq 2$ and $d \neq 4$. However, for $d=4$ we have

$$
f_{1}(a, a) \leqq O(1)|\log a| .
$$

Hence, (i) is also true for $d=4$.

Let

$$
f_{2}\left(a, a^{\prime}\right)=\max _{\substack{x \in a^{\prime} Z^{d} \\ x \in \operatorname{supp}(g)}}\left(a^{\prime d} \sum_{y \in a^{\prime} Z^{d}} g_{y} C^{(a)}(x-y)^{4}\right) .
$$

As in the proof of Theorem 1.1 given in the last section, we can show that there are constants $c_{3}, c_{4} \in(0, \infty)$ such that

$$
c_{3} V\left(a, a^{\prime}\right)^{2} \leqq f_{2}\left(a, a^{\prime}\right) \leqq c_{4} V\left(a, a^{\prime}\right)^{2},
$$

and so

$$
c_{5} a^{\prime d} c^{(a)}(0)^{4} \leqq f_{2}\left(a, a^{\prime}\right) \leqq c_{6} a^{\prime d} C^{(a)}(0)^{4}
$$

for some constant $c_{5}, c_{6} \in(0, \infty)$.

Lemma 3.2. Suppose that $l_{1, i}+l_{2, i}+\sum_{j=i+1}^{k} l_{i, j} \leqq 4$ and $l_{1, i}, l_{2, i}, l_{i, j} \geqq 0$ for $j \in$ $[i+1, k]$. Then there is a constant $c_{7} \in(0, \infty)$ such that

$$
\begin{aligned}
& a^{\prime d} \sum_{x_{i} \in a^{\prime} Z^{d}} g_{x_{i}} \prod_{t=1}^{2} C^{(a)}\left(x_{t}-x_{i}\right)^{l_{t, i}} \prod_{j=i+1}^{k} C^{(a)}\left(x_{i}-x_{j}\right)^{l_{, j}} \\
& \quad \leqq c_{7} f_{2}\left(a, a^{\prime}\right)^{\frac{1}{4}\left(l_{1, i}+l_{2, i}+\sum_{j=t+1}^{k} l_{h, j}\right)}
\end{aligned}
$$

for $x_{1}, x_{2}, x_{i+1}, \ldots, x_{k} \in a^{\prime} Z^{d}$ with $x_{1}, x_{2}, x_{i+1}, \ldots, x_{k} \in \operatorname{supp}(g)$. 
Proof. By the Hölder inequality we know that the left-hand side of (3.2) is less than

$$
\begin{aligned}
O(1) & \prod_{j=1}^{2}\left(\sum_{x \in a^{\prime} Z^{d}, x_{i} \in \operatorname{supp}(g)} C^{(a)}\left(x_{j}-x_{i}\right)^{4}\right)^{\frac{l_{j, l}}{4}} \prod_{j=i+1}^{k}\left(\sum_{x \in a^{\prime} Z^{d}, x_{i} \in \operatorname{supp}(g)} C^{(a)}\left(x_{i}-x_{j}\right)^{4}\right)^{\frac{l_{t, j}}{4}} \\
& \cdot\left(\sum_{x \in a^{\prime} Z^{d}, x_{i} \in \operatorname{supp}(g)} 1\right)^{1-\frac{1}{4}\left(l_{1, i}+l_{2, i}+\sum_{j=l+1}^{k} l_{l, j}\right)} \\
& \leqq O(1) f_{2}\left(a, a^{\prime}\right)^{\frac{1}{4}\left(l_{1, i}+l_{2, l}+\sum_{j=l+1}^{k} l_{l, J}\right)},
\end{aligned}
$$

where we used the fact: $a^{\prime d} \sum_{x_{i} \in a^{\prime} Z^{d}, x_{i} \in \operatorname{supp}(g)} 1 \leqq O(1)$ to obtain the last inequality. This completes the proof of Lemma 3.2.

We now use Lemma 3.1 and Lemma 3.2 to prove Lemma 2.1.

Proof of Lemma 2.1. We will only consider the quantity $q_{a, a^{\prime}}\left(x_{1}, \ldots, x_{k}\right)$ in which there is a pair $(i, j)$ such that $l_{i, j} \in\{1,2,3\}$. If $l_{i, j}=3$, then there must be a new pair $\left(i, j^{\prime}\right)$ or $(h, i)$ such that $l_{i, j^{\prime}}=1$ or $l_{h, i}=1$. Thus we may always assume that there is a pair $(i, j)$ in the quantity $q_{a, a^{\prime}}\left(x_{1}, \ldots, x_{k}\right)$ such that $l_{i, j} \in\{1,2\}$. Without loss of generality we may also assume $l_{1,2} \in\{1,2\}$. Thus, the quantity $q_{a, a^{\prime}}\left(x_{1}, \ldots, x_{k}\right)$ can be expressed as follows:

$$
\begin{aligned}
q_{a, a^{\prime}}\left(x_{1}, \ldots, x_{k}\right)= & C^{(a)}\left(x_{1}-x_{2}\right)^{l_{1,2}} \prod_{j=3}^{k} C^{(a)}\left(x_{1}-x_{j}\right)^{l_{1, j}} \\
& \cdot \prod_{j=3}^{k} C^{(a)}\left(x_{2}-x_{j}\right)^{l_{2, j}} \prod_{i=3}^{k-1} \prod_{j=i+1}^{k} C^{(a)}\left(x_{i}-x_{j}\right)^{l_{i, j}} .
\end{aligned}
$$

We remark again that

$$
\sum_{i=1}^{k-1} \sum_{j=i+1}^{k} l_{i, j}=2 k .
$$

Then, by Lemma 3.2 we know that

$$
\begin{aligned}
& a^{\prime d(k-2)} \sum_{x_{3},, x_{k} \in a^{\prime} Z^{d}} \prod_{i=3}^{k} g_{x_{i}} \prod_{j=3}^{k} C^{(a)}\left(x_{1}-x_{j}\right)^{l_{1, j}} \\
& \quad \cdot \prod_{j=3}^{k} C^{(a)}\left(x_{2}-x_{j}\right)^{l_{2, j}} \prod_{i=3}^{k-1} \prod_{j=i+1}^{k} C^{(a)}\left(x_{i}-x_{j}\right)^{l_{l, j}} \\
& \leqq O(1) f_{2}\left(a, a^{\prime}\right)^{\frac{1}{4}\left(\sum_{i=3}^{k}\left(l_{1, l}+l_{2, l}\right)+\sum_{l=3}^{k} \sum_{j=l+1}^{k} l_{i, J}\right)} \\
& \leqq O(1) f_{2}\left(a, a^{\prime}\right)^{\frac{k}{2}-\frac{1}{4} l_{1,2}}, \quad \forall x_{1}, x_{2} \in a^{\prime} Z^{d}
\end{aligned}
$$

with $x_{1}, x_{2} \in \operatorname{supp}(g)$. Therefore,

$$
\begin{aligned}
& a^{\prime d k} \sum_{x_{1}, x_{k} \in a^{\prime} Z^{d}} \prod_{i=1}^{k} g_{x_{i}} q_{a, a^{\prime}}\left(x_{1}, \ldots, x_{k}\right) \\
& \leqq O(1) f_{2}\left(a, a^{\prime}\right)^{\frac{k}{2}-\frac{1}{4} l_{1,2}} a^{\prime} 2 d \sum_{x_{1}, x_{2} \in a^{\prime} Z^{d}} g_{x_{1}} g_{x_{2}} C^{(a)}\left(x_{1}-x_{2}\right)^{l_{1,2}} .
\end{aligned}
$$


If $d=4$ and $l_{1,2}=2$, by Lemma 3.1 and (3.1) we then know that the right-hand side of (3.3) is less than

$$
\begin{aligned}
& O(1)\left(a^{\prime d} C^{(a)}(0)^{2}+|\log a|\right) f_{2}\left(a, a^{\prime}\right)^{\frac{k}{2}-\frac{1}{4} l_{1,2}} \\
& \quad \leqq O(1)\left(a^{\prime d} C^{(a)}(0)^{2}+|\log a|\right)\left(a^{\prime d} C^{(a)}(0)^{4}\right)^{\frac{k}{4}-\frac{1}{2}} \\
& \quad \leqq O(1) a^{\prime 2}\left(a^{\prime 4} C^{(a)}(0)^{4}\right)^{\frac{k}{2}}+O(1)|\log a| a^{\prime-2} a^{4}\left(a^{\prime 4} C^{(a)}(0)^{4}\right)^{\frac{k}{2}} \\
& \quad \leqq O(1) a^{\prime}\left(a^{\prime 4} C^{(a)}(0)^{4}\right)^{\frac{k}{2}}
\end{aligned}
$$

since $a^{\prime} \geqq a$. If $l_{1,2}=2, d \geqq 2$ and $d \neq 4$, then by Lemma 3.1 and (3.1) again we know that the right-hand side of (3.3) is less than

$$
O(1) a^{\prime d / 2}\left(a^{\prime d} C^{(a)}(0)^{\frac{k}{2}}\right.
$$

However, if $l_{1,2}=1$, by Lemma 3.1 and (3.1) we know that the right-hand side of (3.3) is less than

$$
O(1) a^{\prime 3 d / 4}\left(a^{\prime d} C^{(a)}(0)^{4}\right)^{\frac{k}{2}}
$$

for $d \geqq 2$. Since $\lim _{a \rightarrow 0^{+}} a^{\prime}=0$ and there are constants $c_{8}, c_{9} \in(0, \infty)$ such that

$$
c_{8} a^{\prime d} C^{(a)}(0)^{4} \leqq V\left(a, a^{\prime}\right)^{2} \leqq c_{9} a^{\prime d} C^{(a)}(0)^{4}
$$

we have

$$
\lim _{a \rightarrow 0^{+}} V\left(a, a^{\prime}\right)^{-2} a^{\prime d k} \sum_{x_{1}, x_{k} \in a^{\prime} Z^{d}} \prod_{i=1}^{k} g_{x_{i}} q_{a, a^{\prime}}\left(x_{1}, \ldots, x_{k}\right)=0,
$$

which proves the desired result. This ends the proof of Lemma 2.1.

Acknowledgement We thank Prof. Michael Röckner for very interesting and stimulating discussions. The financial support of SFB 343 in Bielefeld and a DFG research project for the last named author (X Y Zhou) is gratefully acknowledged

Added in proof: This paper was under publication when the second author, Xian Yin Zhou, passed away on April 22, 1996, due to a sudden heart disease He was only 32 years old. We deeply mourn Zhou's departure, a terrible loss for his family, all friends and coworkers, and the whole mathematician community.

\section{References}

[AZ] Albeverio, S, Zhou, X.Y.: A new convergent lattice approximation for the $\phi_{2}^{4}$-quantum field Preprint (1995)

[BFS] Brydges, D., Fröhlich, J., Sokal, A D.: A new proof of the existence and nontrivality of the continuum $\phi_{2}^{4}$ and $\phi_{3}^{4}$ quantum field theories. Commun. Math. Phys 91, 141-186 (1983)

[Fe] Feldman, J.: The $\lambda \phi_{3}^{4}$ field theory in a finite volume. Commun Math. Phys. 37, 93-120 (1974)

[GJ] Glimm, J, Jaffe, A.: Positivity of the $\phi_{3}^{4}$ Hamiltonian Fort der Phys 21, 327-376 (1973)

$[\mathrm{Ne}]$ Newman, C.M.: A general central limit theorem for FKG systems. Commun. Math. Phys. 91, 75-80 (1983)

[P] Park, Y M.: Lattice approximation of the $\left(\lambda \phi^{4}-\mu \phi\right)_{3}$ field theory in a finite volume. J. Math. Phys. 16, 1065-1075 (1975)

[Si] Simon, B.: The $P(\phi)_{2}$ Euclidean (Quantum) Field Theory. Princeton, NJ: Princeton University Press, 1974 